\title{
4-D PET-MR with Volumetric Navigators and Compressed Sensing
}

\section{Pedemonte, Stefano ; Catana, Ciprian; Van Leemput, Koen}

\section{Published in:}

Computational Methods for Molecular Imaging

Link to article, DOI:

10.1007/978-3-319-18431-9_10

Publication date:

2015

Document Version

Peer reviewed version

Link back to DTU Orbit

\section{Citation (APA):}

Pedemonte, S., Catana, C., \& Van Leemput, K. (2015). 4-D PET-MR with Volumetric Navigators and Compressed Sensing. In F. Gao, K. Shi, \& S. Li (Eds.), Computational Methods for Molecular Imaging (pp. 93101). Springer. Lecture Notes in Computational Vision and Biomechanics No. 22 https://doi.org/10.1007/978-3319-18431-9_10

\section{General rights}

Copyright and moral rights for the publications made accessible in the public portal are retained by the authors and/or other copyright owners and it is a condition of accessing publications that users recognise and abide by the legal requirements associated with these rights.

- Users may download and print one copy of any publication from the public portal for the purpose of private study or research.

- You may not further distribute the material or use it for any profit-making activity or commercial gain

- You may freely distribute the URL identifying the publication in the public portal 


\title{
4-D PET-MR with Volumetric Navigators and Compressed Sensing
}

\author{
Stefano Pedemonte ${ }^{12}$, Ciprian Catana $^{1}$, Koen Van Leemput ${ }^{123}$ \\ 1 Athinoula A. Martinos Center for Biomedical Imaging, MGH/Harvard, MA, USA \\ 2 Department of Information and Computer Science, Aalto University, FI \\ 3 Department of Applied Mathematics and Computer Science, \\ Technical University of Denmark, DE
}

\begin{abstract}
Hybrid PET-MR scanners acquire multi-modal signals simultaneously, eliminating the requirement of software alignment between the MR and PET imaging data. However, the acquisition of high-resolution $\mathrm{MR}$ and PET images requires long scanning times, therefore movement of the subject during the acquisition deteriorates both the PET and the MR images. In this work we have developed an approach for tightly integrated PET-MR imaging, making use of volumetric MR navigators to inform, in real-time, both the MR acquisition and the PET reconstruction. The integrated imaging procedure that we describe exploits the simultaneity of MR and PET in hybrid PET-MR systems, producing inherently-aligned motion-free MR and PET images. We describe the system setup, the algorithm for motion-corrected reconstruction, an adaptive sinogram binning algorithm and software design decisions aimed at integrating tightly the MR and PET subsystems. Application of the integrated motion-corrected acquisition procedure to a phantom study and to a volunteer subject demonstrates the validity of the approach for a variety of motion patterns.
\end{abstract}

\section{Introduction}

Hybrid PET-MR scanners acquire multi-modal signals simultaneously, eliminating the requirement of software alignment between the MR and PET imaging data. However, the acquisition of high-resolution MR and PET images requires long scanning times. Movement of the subject during the acquisition determines a deterioration of both the PET and the MR images. In the context of neuroimaging, recent advancements of MR technology have seen the development of prospective motion correction algorithms [1] [2] based on the insertion, in the MR sequences, of navigators designed to acquire information about the position of the head. In this work we have developed an approach for tightly integrated PET-MR imaging, making use of navigators to inform both the MR acquisition and the PET reconstruction. The integrated imaging procedure that we describe exploits the simultaneity of PET and MR, producing inherently-aligned motionfree PET and MR images. The methodology that we describe is based on a spatio-temporal model of PET imaging that enables the inclusion of sparsity 
constraints. We describe a reconstruction algorithm based on the Alternating Direction Method of Multipliers that enables the use of the non-smooth sparsity prior while incorporating efficiently the information from multiple time frames.

\section{Method}

\subsection{Volumetric navigators}

High resolution tissue contrast images are acquired with the MEMPRAGE sequence, widely employed for morphometry studies. In order to acquire motion information, sampling of k-space is interleaved with the acquisition, during the dead time of each repetition (TR) of a volumetric navigator (vNav) [2]. The vNav consists of a $32^{3}$ isotropic volume 3D-encoded with EPI, requiring $275 \mathrm{~ms}$ to acquire. Motion estimation is performed using the PACE [3] library to register each vNav's image to the reference vNav acquired in the first TR. Due to the low resolution of the vNav image, this is performed reliably in under $100 \mathrm{~ms}$ on current scanner hardware (Siemens Biograph mMR). The direction of the MR gradient for the acquisition of each new slice of $k$-space for the MEMPRAGE sequence is adjusted in real-time according to the pose estimate provided by the vNav, effectively acquiring all of $\mathrm{k}$-space in the moving frame attached to the head of the subject.

\subsection{Compressive model-based motion correction}

The PET list-mode data is binned into $N_{t}$ sinograms $q_{d}^{t}$, with $t=1,2, \ldots, N_{t}$ indexing the time frames and $d$ indexing the lines-of-response (LOR) of the scanner. Let $p_{v d}$ be the probability that a decay event at location $X_{v}$ within the imaging volume of the scanner is detected in LOR $d$; let $L^{t}$ be the $[4 \times 4]$ transformation matrix that maps the coordinate system attached to the head of the patient to the coordinate system of the PET scanner and let $\widetilde{L}^{t}$ be the corresponding $\left[N_{v} \times N_{v}\right]$ resampling operator, with $N_{v}$ voxels of the imaging volume. The model is framed in the probabilistic setting. In order to account for motion, in this first formulation, we introduce the assumption that the activity in the coordinate system attached to the head of the patient is constant: $\boldsymbol{\lambda}=\lambda_{1}, \ldots, \lambda_{v}, \ldots, \lambda_{N_{v}}$. Under this assumption, the conditional probability distribution associated to the number of counts in LOR $d$ at time $t$ is given by:

$$
p\left(q_{d}^{t} \mid \boldsymbol{\lambda}, \widetilde{L}^{t}\right)=\mathcal{P}\left(\sum_{v} p_{v d}\left[\widetilde{L}^{t} \boldsymbol{\lambda}\right]_{v} ; q_{d}^{t}\right)
$$

We assume a sparsifying L1-norm prior probability distribution for the activity:

$$
p(\boldsymbol{\lambda}) \propto e^{-\beta\|\boldsymbol{\lambda}\|_{1}}
$$




\subsection{Alternating Direction Method of Multipliers}

The most commonly employed algorithm for PET reconstruction is Maximum Likelihood Expectation Maximization - MLEM (and its variant OSEM). Compared to other optimization algorithms, MLEM has the advantage of not requiring the selection of the step size, providing a simple and reliable solution. However MLEM is only applicable to the unconstrained reconstruction (see e.g. [4]). Although approximations of MLEM have been devised to include differentiable constraints, there use of a non-differentiable prior such as the sparsity prior (2) poses an additional challenge. Here, in order to include the sparsity prior, we adopt a data-augmentation method, the Alternating Direction Method of Multipliers (ADMM). As discussed in the review of ADMM presented in [5], in order to derive the ADMM update formula, for the maximum probability problem, we reformulate the problem as a constrained linear program with equality constraints:

$$
\begin{array}{r}
\hat{\boldsymbol{\lambda}}=\arg \min -\sum_{t} \log p\left(q_{d}^{t} \mid \boldsymbol{\lambda}_{t}, \widetilde{L}^{t}\right)-\log p(\boldsymbol{\lambda}) \\
\text { subject to } \boldsymbol{\lambda}_{t}=\boldsymbol{\lambda}, \text { for each } t
\end{array}
$$

The point is that the variables $\boldsymbol{\lambda}_{i}$ must ultimately equal each other, but they can temporarily be unequal while they separately try to satisfy different cost functions. This optimization problem corresponds to the maximization of the augmented Lagrangian:

$$
\begin{aligned}
L\left(\boldsymbol{\lambda}, \boldsymbol{\lambda}_{1}, \ldots, \boldsymbol{\lambda}_{t}, u_{1}, \ldots, u_{t}\right) & =-\sum_{t} \log p\left(q_{d}^{t} \mid \boldsymbol{\lambda}_{t}, \widetilde{L}^{t}\right)-\log p(\boldsymbol{\lambda})+ \\
& +\sum_{t} u_{t}^{T}\left(\boldsymbol{\lambda}-\boldsymbol{\lambda}_{t}\right)+\frac{\rho}{2} \sum_{t}\left\|\boldsymbol{\lambda}-\boldsymbol{\lambda}_{t}\right\|_{2}^{2},
\end{aligned}
$$

where $u_{t}$ are the Lagrange multipliers and $\frac{\rho}{2} \sum_{t}\left\|\boldsymbol{\lambda}-\boldsymbol{\lambda}_{t}\right\|_{2}^{2}$ is the augmentation term. Minimization of the augmented Lagrangian with dual descent yields the ADMM algorithm [5]:

$$
\begin{aligned}
\boldsymbol{\lambda}_{t}^{n+1} & :=\arg \min _{v}-\log p\left(q_{d}^{t} \mid v, \widetilde{L}^{t}\right)+\frac{\rho}{2}\left\|v+u_{t}^{n}-\boldsymbol{\lambda}^{n}\right\|_{2}^{2} \\
\boldsymbol{\lambda}^{n+1} & :=S_{\frac{\rho}{\beta}}\left(\frac{1}{N_{t}} \sum_{t} \boldsymbol{\lambda}_{t}^{n+1}+\frac{1}{\rho N} \sum_{t} u_{t}^{n}\right) \\
u_{t}^{n+1} & :=u_{t}^{n}+\boldsymbol{\lambda}_{t}^{n+1}-\boldsymbol{\lambda}^{n+1},
\end{aligned}
$$

where $S_{\frac{\rho}{\beta}}$ is the soft-thresholding operator (see [5]). For the update of $\boldsymbol{\lambda}_{t}^{n+1}$ we adopt a single iteration of OSEM with warm start (i.e. initializing $v$ at $\boldsymbol{\lambda}_{t}^{n}$ ) and with the One Step Late approximation proposed by Green [4]. In order to account for scatter and randoms, we adopt the Ordinary Poisson version, obtaining:

$$
\lambda_{t, v}^{n+1}=\lambda_{t, v}^{n}\left[\widetilde{L}^{t}\right]^{T}\left[\frac{1}{\sum_{d \in \mathcal{D}^{t}} p_{v d}-\rho\left(\boldsymbol{\lambda}_{t}^{n}+u_{t}^{n}-\boldsymbol{\lambda}^{n}\right)} \sum_{d \in \mathcal{D}^{t}} \frac{p_{v d} q_{d}^{t}}{\sum_{v^{\prime}} p_{v^{\prime} d}\left[\widetilde{L}^{t} \lambda\right]_{v^{\prime}}+r_{d}^{t}+s_{d}^{t}}\right],
$$


where $\mathcal{D}^{t}$ are time-dependent subsets of the LORs of the scanner. In the experiments that follow, time-dependent randoms rates $r_{d}^{t}$ are estimated from single crystal event rates obtained by instantiating singles sinograms for each time frame; the scatter estimates $s_{d}^{t}$ are obtained by weighting, by the duration of the time frames, the scatter estimate obtained by single scatter simulation (SSS) [6] based on the static, non motion-corrected, reconstruction. The estimate of the attenuation map is derived from a radial-VIBE MR image acquired right before the first TR of the vNav MEMPRAGE sequence. As dictated by the model, projections and back-projections account for the attenuation by transforming the attenuation map by $\widetilde{L}^{t}$ at each time frame.
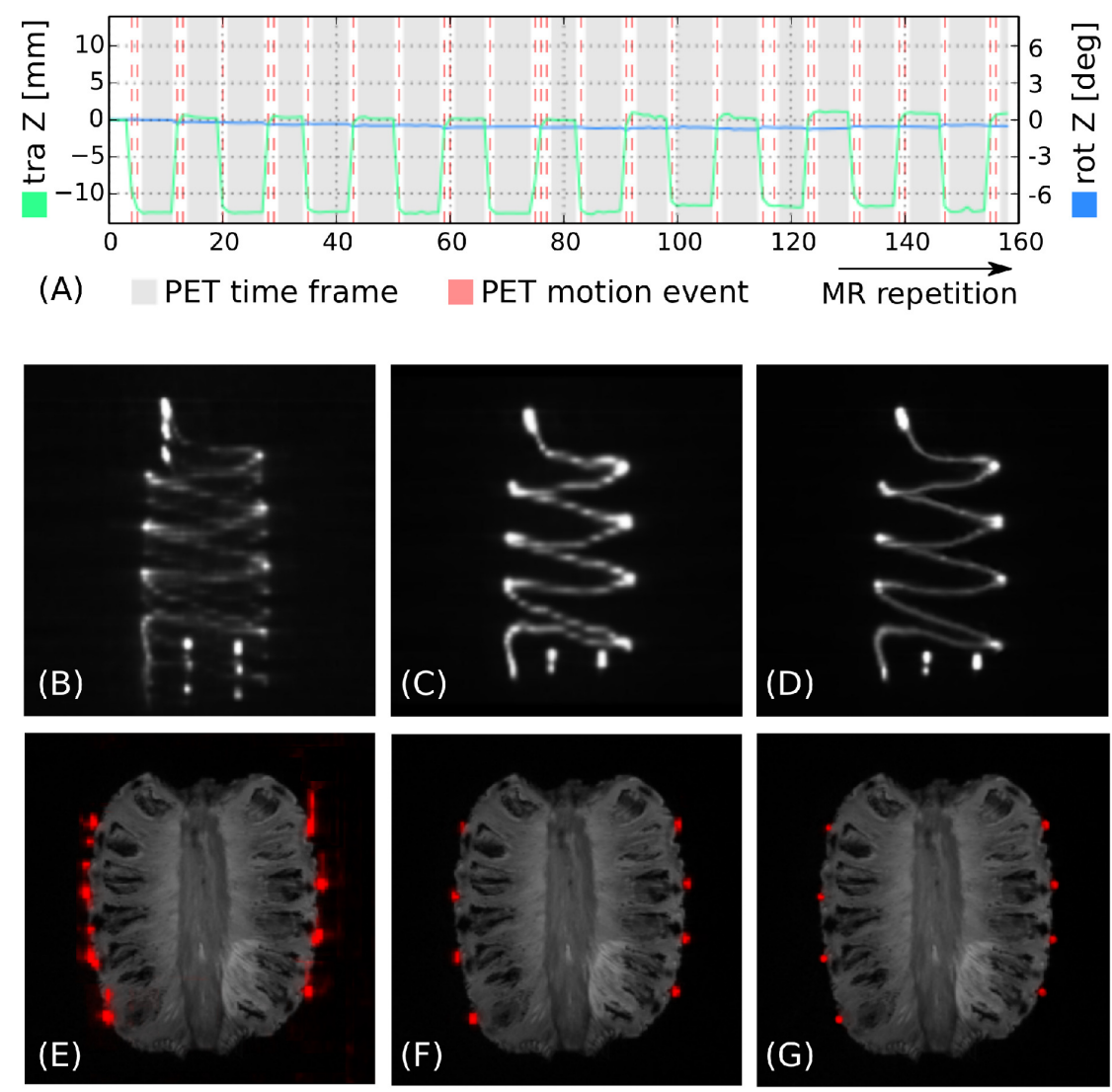

Fig. 1. Pineapple with helicoidal FDG capillary source. (A) Estimated motion profiles (tanslation along the axis of the scanner $\mathrm{Z}$ and rotation around $\mathrm{Z}$ ). Each gray box corresponds to a sinogram. (B-C-D) Volume renderings of the PET reconstructions without motion correction (B), with motion correction (C), with motion correction and sparsity (D). (E-F-G) Representative slice of fused MR and PET images without motion correction (E), with motion correction $(\mathrm{F})$, with motion correction and sparsity constraint $(\mathrm{G})$. 


\subsection{Extraction of the motion events}

In order to optimize the computing resources, binning of the list-mode data into the 4 -D sinogram $q_{d}^{t}$ is performed adaptively. Let us index with $t^{\prime}=1,2, \ldots$ the MR repetitions and denote by $M^{t^{\prime}}$ the $[4 \times 4]$ transformation matrix that maps the vNav at time $t^{\prime}$ to the first vNav. After each vNav acquisition and registration, at time $t^{\prime}$, the binning algorithm decides whether a motion event has occurred by setting a threshold on the extent of motion since the previous motion event $t^{\prime *}$ (starting with $t^{\prime *}=1$ ). As the measure of the extent of motion, we employ the mean voxel displacement in the imaging volume:

$$
w^{t^{\prime}}=\frac{1}{N_{v}} \sum_{v=1}^{N_{v}}\left\|M^{t^{\prime *}} X_{v}-M^{t^{\prime}} X_{v}\right\|
$$

Each time a motion event is detected, the PET time frame index $t$ is increased; a new sinogram is instantiated and $L^{t}$ is set to $L^{t}=M^{t^{\prime}} M^{\mathrm{MR} \rightarrow \mathrm{PET}}$, where the calibration matrix $M^{\mathrm{MR} \rightarrow \mathrm{PET}}$ is computed initially according to the $\mathrm{MR}$ acquisition settings, the geometry of the system and the position of the bed of the scanner. In the current implementation, interaction events acquired during the period TR preceding each motion event are discarded.

\subsection{Software framework for 4-D reconstruction}

A considerable software design effort was necessary in order to implement the 4-D reconstruction algorithm that processes the list-mode data, synchronizing the MR and PET subsystems. Projection and back-projection algorithms based on ray-tracing have been designed with the aim of computing, without overhead, the spatial transformations required by the algorithm of eq. (10). The projection and back-projection algorithms operate directly on compressed 4-D sinograms designed to enable storage of the full sequence on the memory of a single Graphics Processing Unit (GPU).

\section{Experiments}

Experiments were performed on a Siemens scanner Biograph mMR. TR was set to $2.5 \mathrm{sec}$ and the threshold of $w^{t^{\prime}}$ was set to $0.5 \mathrm{~mm}$.

Phantom study - A pineapple with attached a helicoidal capillary filled with 1.5 $\mathrm{mCi}$ of FDG was scanned for $400 \mathrm{sec}$. The phantom was translated periodically by $12 \mathrm{~mm}$ along the axis of the scanner every $40 \mathrm{sec}$. Fig. 1 (A) reports the motion estimates. Fig. 1 (B-G) display the reconstructions with and without motion correction and with and without sparsity constraint (sparsity parameter $\beta=0.1)$.

Healthy volunteer - A healthy volunteer was scanned for $400 \mathrm{sec}, 20 \mathrm{~min}$ post injection of $4.7 \mathrm{mCi}$ of FDG. Fig. 2 reports the motion estimates, the extent of motion, the motion events, and the unconstrained reconstructions without motion correction and the constrained motion corrected reconstruction. The subject 

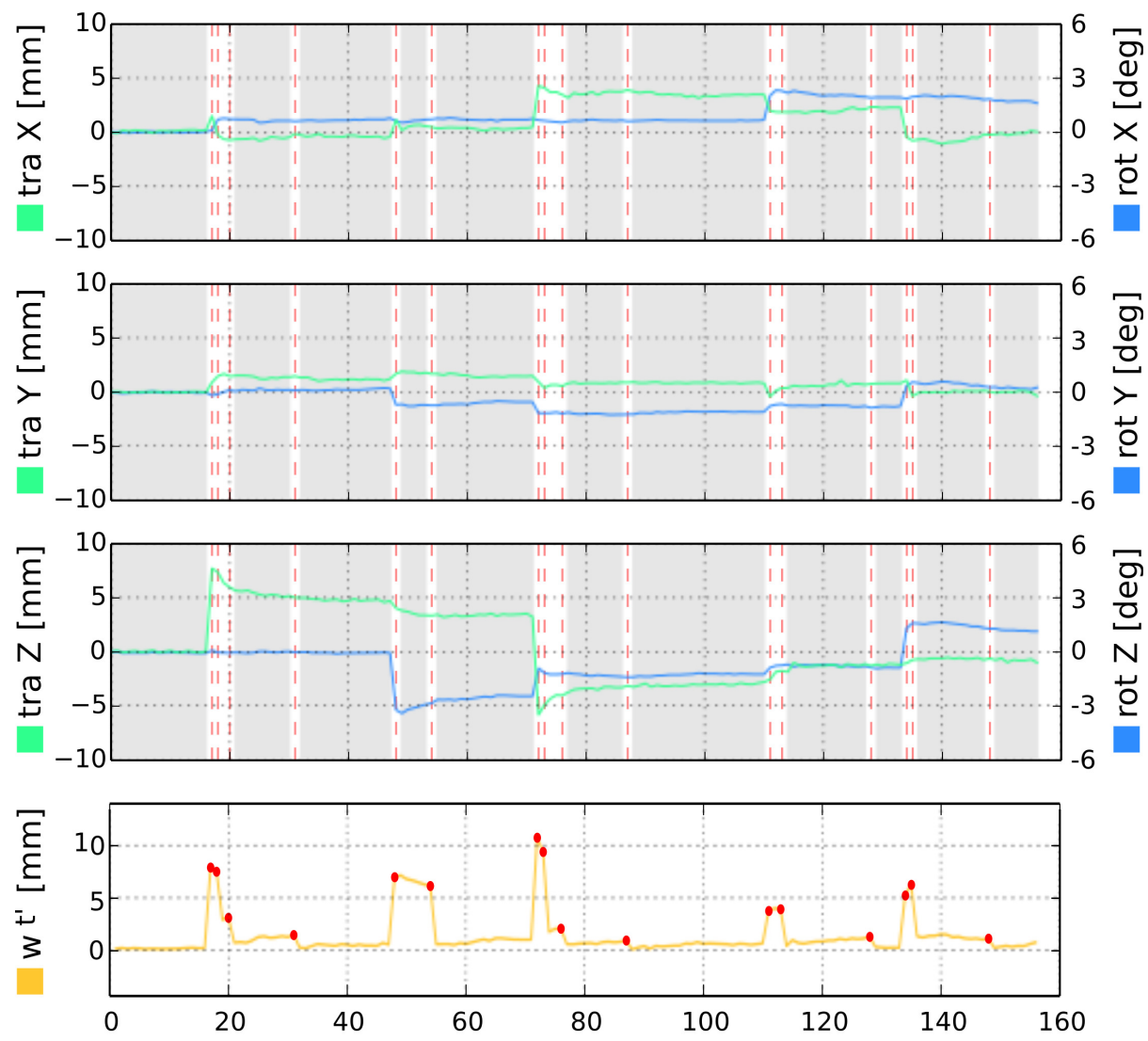

(A) PET time frame $\quad$ PET motion event $\overrightarrow{M R \text { repetition }}$
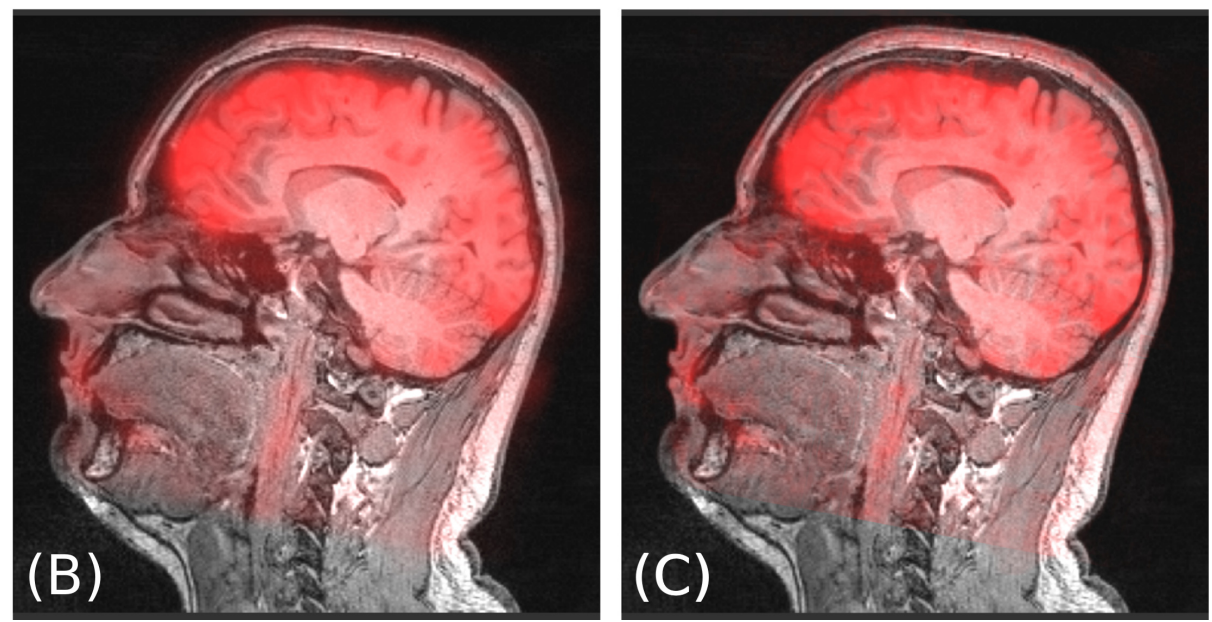

Fig. 2. Healthy volunteer. (A) Estimated motion profiles and extent of motion $w^{t^{\prime}}$. (B)(C) Representative slices of the fused MR and PET images without motion correction (B) and with motion correction and sparsity constraint (C). 

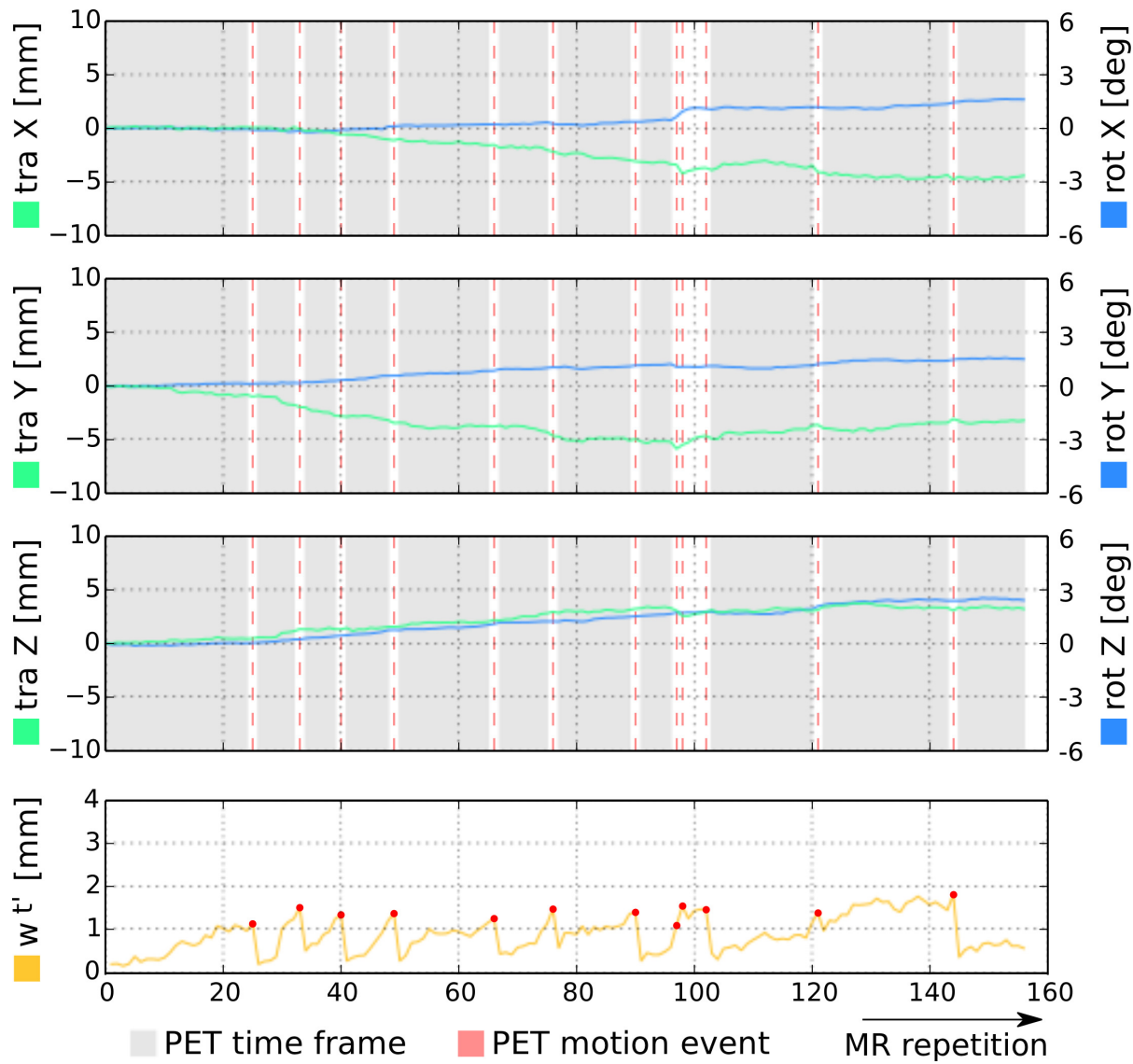

Fig. 3. Healthy volunteer on $3 \mathrm{~cm}$-thick latex pillow. The adaptive binning algorithm produces sinograms with longer duration (gray boxes) when the pillow is fully compressed. 
was scanned again for $400 \mathrm{sec}$ after insertion of a $3 \mathrm{~cm}$-thick latex pillow. Note that in case of continuous motion, as displayed in Fig. 3, the algorithm of paragraph (2.4) adapts the duration of the sinograms according to the rate of change of the extent of motion. Note, in Fig. 2 and Fig. 3, that the binning algorithm generated, in both experiments, 11 time frames. The reconstruction time, which scales linearly with the number of time frames, was 14 minutes.

\section{Conclusion}

We have developed an integrated approach to PET-MR that enables the reconstruction of motion-free PET and MR images in the same frame of reference. The model-based approach for PET reconstruction that we have described enables us to account for attenuation, randoms and scatter, while integrating motion information. The event-based methodology based on a relative measure of extent of motion enables the correction of large motion events and of slow drifts, adapting the demand of computing resources to the motion patterns. The optimization algorithm, based on the Alternating Direction Method of Multipliers, enables the inclusion of a sparsity constraint, which improves image quality. The GPU accelerated software implementation (http://niftyrec.scienceontheweb.net) enables reconstruction times comparable to static reconstruction. In future work we will explore the inclusion of pharmacokinetics in the imaging model, the acquisition of multiple MR sequences with embedded volumetric navigators and non-rigid motion.

\section{Acknowledgements}

This research was supported by the NIH NCRR (P41-RR14075), the NIH NIBIB (R01EB013565), and TEKES (ComBrain).

\section{References}

1. Van der Kouwe et al. Real-time rigid body motion correction and shimming using cloverleaf navigators. MRM, 56(5):1019-32, 2006.

2. Tisdall et al. Volumetric navigators for prospective motion correction and selective reacquisition in neuroanatomical MRI. MRM, 68(2):389-99, 2012.

3. Thesen et al. Prospective acquisition correction for head motion with image-based tracking for real-time fMRI. Magn. Reson. Med., 44(3):457-65, 2000.

4. P.J. Green. Bayesian Reconstructions From Emission Tomography Data Using a Modified EM Algorithm. IEEE TMI, 9(1):84-93, 1990.

5. S. Boyd, N. Parikh, E. Chu, B. Peleato, and J. Eckstein. Distributed Optimization and Statistical Learning via the Alternating Direction Method of Multipliers. Foundations and Trends in Machine Learning, 3(1):1-122, 2012.

6. Watson et al. A Single Scatter Simulation Technique for Scatter Correction in 3D PET. Three-Dimensional Image Reconstruction in Radiology and Nuclear Medicine, 4:255-68, 1996. 